\title{
A New Result on Tricyclic Mappings
}

\author{
Taoufik Sabar, Abdelhafid Bassou $(\mathbb{D}$, and Mohamed Aamri $\mathbb{1 0}$ \\ Laboratory of Algebra, Analysis and Applications(L3A), Department of Mathematics and Computer Science, \\ Faculty of Sciences Ben M'Sik, Hassan II University of Casablanca, P. B. 7955 Sidi Othmane, Casablanca, Morocco
}

Correspondence should be addressed to Abdelhafid Bassou; hbassou@gmail.com

Received 8 July 2020; Revised 14 August 2020; Accepted 24 August 2020; Published 5 October 2020

Academic Editor: Niansheng Tang

Copyright (C) 2020 Taoufik Sabar et al. This is an open access article distributed under the Creative Commons Attribution License, which permits unrestricted use, distribution, and reproduction in any medium, provided the original work is properly cited.

In this note, we present a best proximity point theorem for tricyclic contractions in the setting of CAT(0) spaces. In the same context, we give an elaborate counterexample.

\section{Introduction and Preliminaries}

Back in 2017, the current authors introduced the class of tricyclic mappings and best proximity points thereof. A mapping $T: A \cup B \cup C \longrightarrow A \cup B \cup C$ is said to be tricyclic provided that $T(A) \subseteq B, T(B) \subseteq C$, and $T(C) \subseteq A$, where $A, B$, and $C$ are nonempty subsets of a metric space $(X, d)$, and a best proximity point of $T$ is a point $x \in A \cup B \cup C$ such that $D\left(x, T x, T^{2} x\right)=$ $D(A, B, C)$ where the mapping $D: X \times X \times X \longrightarrow[0,+\infty)$ is defined by $D(x, y, z):=d(x, y)+d(y, z)+d(z, x)$ and $D$ $(A, B, C):=\inf \{D(x, y, z): x \in A, y \in B$ and $z \in C\}$. Moreover, the mapping $T$ is said to be a tricyclic contraction if it is tricyclic and verifies $D(T x, T y, T z) \leq k D(x, y, z)+(1-k) \delta$ $(A, B, C)$, for some $k \in(0,1)$ and for all $(x, y, z) \in A \times B \times C$. For detailed information, we refer to $[1,2]$.

In the same research paper where they defined tricylic mappings, the following best proximity point result was obtained.

Theorem 1 (see [1]). Let $A, B$, and $C$ be nonempty, closed, bounded, and convex subsets of a reflexive Banach space $X$, and let $T: A \cup B \cup C \longrightarrow A \cup B \cup C$ be a tricyclic contraction map. Then, $T$ has a best proximity point. we set

Given three subsets $A, B$, and $C$ of a metric space $(X, d)$,

$$
E=:\left\{(x, y) \in A \times B: \Delta_{(x, y)}(C) \leq \lambda\right\},
$$

where $\Delta_{(x, y)}(C):=\sup \{D(x, y, z): z \in C\}$ and $\lambda$ is a nonnegative real number.

Taking heed in the proof of the main result of [1], one becomes aware that the proof of such result in a metric space, where some sort of convexity and reflexivity is defined of course, comes down to proving that the setting $E$ is convex. It is easy to see that $E$ is convex in linear normed spaces. But how far from linear metric spaces can we go? Later on, we show that the set $E$ loses its convexity in an arbitrary geodesic metric space.

In this paper, we extend the previous theorem to a wider class of spaces, and it is that of geodesic metric spaces. Then, we give an answer to the tricyclic mapping-related question that remained open for a couple of years in the form of a counterexample.

Preparatory to giving our main result, we hark back to geodesic spaces and one of their most important subclasses CAT (0) spaces.

A geodesic space $(X, d)$ is a metric space in which every two points $x$ and $y$ are joined by a geodesic, that is, a map $c:[0, l] \longrightarrow X$ such that $c(0)=x$ and $c(l)=y$ and $d\left(c(t), c\left(t^{\prime}\right)\right)=\left|t-t^{\prime}\right|$ for all $t, t^{\prime} \in[0, l]$. A subset $C$ of a geodesic space $X$ is said to be convex if every pair of points $x, y \in C$ are joined by a geodesic whose image is contained in $C$. A point $z \in X$ belongs to a geodesic segment $[x, y]$ if and only if there exists $\lambda \in[0,1]$ such that $d(x, z)=\lambda d(x, y)$ and $d(y, z)=(1-\lambda) d(x, y)$ and it is written as $z=(1-t) x \oplus t y$. 
The geodesic space $X$ is said to be reflexive if for every decreasing chain $\left(C_{i}\right) \subset X$, where $i \in I, C_{i}$ is nonempty, bounded, closed, and convex for all $i \in I$ such that we have $\cap_{i \in I} C_{i} \neq \varnothing$.

A geodesic triangle $\Delta(p, q, r)$ in $(X, d)$ consists of three points $p, q$, and $r$ (vertices of $\Delta$ ) connected by three geodesic segments (sides of $\Delta)$. A comparison triangle for $\Delta(p, q, r)$ is a triangle $\bar{\Delta}(p, q, r):=\Delta(\bar{p}, t \bar{q} n, q \bar{r})$ in the Euclidean plane with the same lengths of sides.

We also recall that a geodesic space is called convex in the sense of Buesmann [3] if given any pair of geodesics $c_{1}:\left[0, a_{1}\right] \longrightarrow X$ and $c_{2}:\left[0, a_{2}\right] \longrightarrow X$; the following inequality is satisfied:

$$
\begin{gathered}
d\left(c_{1}\left(t a_{1}\right), c_{2}\left(t a_{2}\right)\right) \leq(1-t) d\left(c_{1}(0), c_{2}(0)\right) \\
+t d\left(c_{1}\left(a_{1}\right), c_{2}\left(a_{2}\right)\right) \quad \text { for all } t \in[0,1] .
\end{gathered}
$$

Equivalently,

$$
\begin{aligned}
& d\left((1-t) x_{1} \oplus t x_{2},(1-t) y_{1} \oplus t y_{2}\right) \leq(1-t) d\left(x_{1}, y_{1}\right) \\
& \quad+t d\left(x_{2}, y_{2}\right)
\end{aligned}
$$

for all $x_{1}, x_{2}, y_{1}, y_{2} \in X$ and $t \in[0,1]$.

Besides Busemann convex spaces, CAT(0) spaces is a very important subclass of geodesic spaces. Those are metric spaces that are, of course, geodesically connected, and their geodesic triangles are at least as "thin" as their comparison triangles in the Euclidean plane.

Needless to say, CAT (0) spaces are convex in the sense of Busemann. As a matter of fact, that is the most fundamental of the properties defining the nature of CAT(0) spaces. Complete CAT (0) spaces are called Hadamard spaces and are reflexive [4].

\section{Main Results}

Direct usage of the norm properties shows that $E$ is convex if $X$ is a normed linear space and the subsets $A$ and $B$ are convex. The same goes for CAT(0) spaces.

Lemma 1. If $A$ and $B$ are nonempty convex subsets of $a$ $C A T(0)$ space $X$, then so is $E$.

Proof. Let $\left(x_{1}, y_{1}\right),\left(x_{2}, y_{2}\right) \in E$, and the map given by

$$
t \mapsto\left((1-t) x_{1} \oplus t x_{2},(1-t) y_{1} \oplus t y_{2}\right)
$$

is a geodesic joining $\left(x_{1}, y_{1}\right)$ and $\left(x_{2}, y_{2}\right)$ in the set $X \times X$ endowed with the metric $d\left(\left(x, x^{\prime}\right),\left(y, y^{\prime}\right)\right)=d(x, y)+d\left(x^{\prime}, y^{\prime}\right)$, so we must verify that

$$
\left((1-t) x_{1} \oplus t x_{2},(1-t) y_{1} \oplus t y_{2}\right) \in E \text {. }
$$

Given $z \in C$,

$$
\begin{aligned}
& D\left((1-t) x_{1} \oplus t x_{2},(1-t) y_{1} \oplus t y_{2}, z\right) \\
& =d\left((1-t) x_{1} \oplus t x_{2},(1-t) y_{1} \oplus t y_{2}\right) \\
& +d\left((1-t) x_{1} \oplus t x_{2}, z\right)+d\left((1-t) y_{1} \oplus t y_{2}, z\right) \text {, } \\
& \leq(1-t) d\left(x_{1}, y_{1}\right)+t d\left(x_{2}, y_{2}\right)+(1-t) d\left(x_{1}, z\right) \\
& +t d\left(x_{2}, z\right)+(1-t) d\left(y_{1}, z\right)+t d\left(y_{2}, z\right) \text {, } \\
& =(1-t) D\left(x_{1}, y_{1}, z\right)+t D\left(x_{2}, y_{2}, z\right) \\
& \leq \max \left\{D\left(x_{1}, y_{1}, z\right), D\left(x_{2}, y_{2}, z\right)\right\} \\
& \leq \max \left\{\Delta_{\left(x_{1}, y_{1}\right)}(C), \Delta_{\left(x_{2}, y_{2}\right)}(C)\right\} \leq \lambda \text {. }
\end{aligned}
$$

Hence, $E$ is convex.

Now, we can assert our best proximity point existence result.

Theorem 2. Let $A, B$, and $C$ be nonempty, closed, bounded, and convex subsets of Hadamard space $X$, and let $T: A \cup B \cup C \longrightarrow A \cup B \cup C$ be a tricyclic contraction map. Then, $T$ has a best proximity point.

Evidently, the previous theorem generalizes the one of [1], since the latter was proven in the setting of reflexive Banach spaces.

Furthermore, it is easy to see that $E$ is also convex in the setting of hyperbolic spaces in the sense of Kohlenbach [5]. And, in [2], it is proved that $E$ is convex in the setting of $(S)$ - convex metric spaces, if $A, B$, and $C$ verify supplementary conditions. Now, besides the metric spaces mentioned previously in this article, does $E$ stay convex in a more general framework, say, geodesic spaces or any metric spaces, with some sense of convexity?

Next, we claim that it is not the case and we give a subtle counterexample. First, we mention a couple of propositions.

Proposition 1 (see [6], Exercise 4.3.8). The geodesic space $(X, d)$ is of nonnegative (resp., nonpositive) curvature if the following property is true: let $\Delta(p, q, r)$ be a triangle in $X$ and $\Delta(\bar{p}, t \bar{q} n, q \bar{r})$ be its comparison triangle. Then, for points $x$ and $y$ in the sides $[p, q]$ and $[q, r]$ and points $\bar{x}$ and $\bar{y}$ in the sides $[\bar{p}, t \bar{q}]$ and $[\bar{q}, t \bar{r}]$ such that $d(x, q)=d(\bar{x}, t \bar{q})$ and $d(y, q)=d(\bar{y}, t \bar{q})$, the inequality $d(x, y) \geq d(\bar{x}, t \bar{y})$ (resp., $d(x, y) \leq d(\bar{x}, t \bar{y}))$ holds.

The following observation is natural, yet it has been very useful to our work.

Proposition 2. Let $X$ be a space with nonnegative curvature, given any pair of geodesics $c:[0,1] \longrightarrow X$ and $c^{\prime}:[0,1] \longrightarrow X$ emanating from the same point $p=c(0)=c^{\prime}(0)$; the following inequality holds for all $t \in[0,1]:$

$$
d\left(c(t), c^{\prime}(t)\right) \geq t d\left(c(1), c^{\prime}(1)\right)
$$


Proof. Consider a comparison triangle $\bar{\Delta} \subset E^{2}$ for the triangle $\Delta(p, q, r):=\Delta\left(c(0), c(1), c^{\prime}(1)\right)$. Given $t \in[0,1]$, elementary geometry tells us that

$$
d\left(\overline{c(t)}, \overline{t c^{\prime}(t)}\right)=t d\left(\overline{c(1)}, \overline{c^{\prime}(1)}\right)=t d\left(c(1), c^{\prime}(1)\right) .
$$

And, by the nonnegativity of the curvature

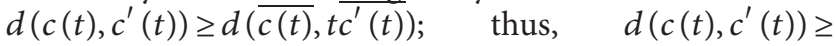
$t d\left(c(1), c^{\prime}(1)\right)$.

Remark 1. The inequality from the previous proposition implies that

$$
\sup _{0<t<1} d\left(c(t), c^{\prime}(t)\right) \geq d\left(c(1), c^{\prime}(1)\right) .
$$

and, inspired by the last inequality, we may claim that there are cases where the inequality in the last proposition becomes

$$
d\left(c(t), c^{\prime}(t)\right)>d\left(c(1), c^{\prime}(1)\right),
$$

for some $t \in(0,1)$, just like illustrated in the following (Figure 1).

Now, let $(X, d)$ be a space with nonnegative curvature, and assume there exist three geodesic segments of $X$, $A=[p, q], \quad B=[p, r], \quad$ and $C=[p, s]$ such that $d(p, q)=d(p, r)=d(s, q)=d(s, r)$ and $d(p, s)=d(q, r)$. Suppose

$$
\Delta_{(q, r)}(C)=D(p, q, r)=D(s, q, r) .
$$

And, from the previous remark, we assume that there exist $x \in(p, q)$ and $y \in(p, r)$ such that

$$
d(x, y)>d(q, r) \text { and } d(x, s)=d(y, s) .
$$

Put

$$
E=\left\{(x, y) \in A \times B: \Delta_{(x, y)}(C) \leq D(p, q, r)\right\} .
$$

Since $(p, p),(q, r) \in E$, if $E$ is convex, then it must contain $(x, y)$. We now show the opposite, in this order, and we suppose that $x$ and $y$ verify the extra condition that, if $d(s, q)>d(s, x)$, we have

$$
d(x, y)-d(q, r)>2(d(s, q)-d(s, x)) .
$$

Then,

$$
\begin{aligned}
& D(x, y, s)=d(x, y)+2 d(s, x), \\
&>d(q, r)+2 d(s, q), \\
&=D(q, r, s)=D(q, r, p) .
\end{aligned}
$$

which means that $E$ is not convex.

Now, we support our claim with a concrete example where all the previous suppositions hold true.

Example 1. Let $X:=S^{2}$ be the unit sphere in $\mathbb{R}^{3}$ endowed with the function $d: X \times X \longrightarrow \mathbb{R}$ that assigns to each pair $(x, y) \in X \times X$ the unique real number $d(x, y)=$

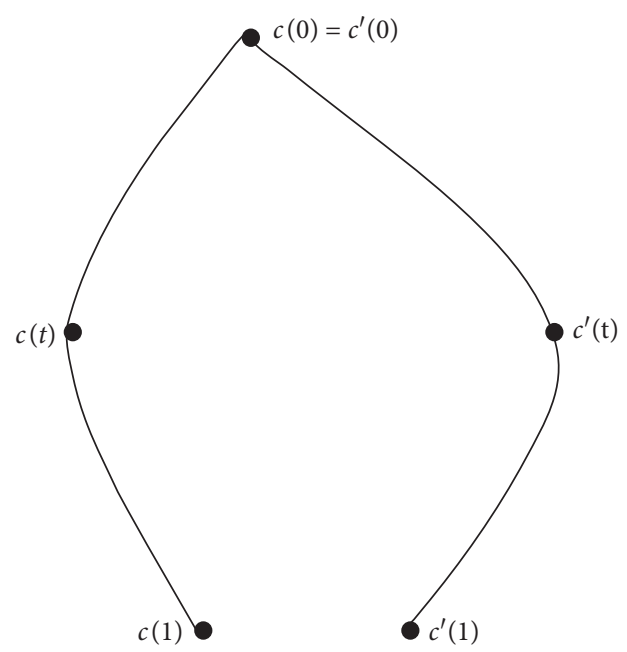

Figure 1: An illustrative figure.

$\cos ^{-1}(\langle x, y\rangle) \in[0, \pi]$ where $\langle x, y\rangle$ denotes the Euclidean scalar product. It is known that $d$ is a metric on a $X$. Moreover, $d(x, y)$ is the length of the shortest curve on $S^{2}$ connecting $x$ and $y$. Such a curve is a segment on a great circle through $x$ and $y$ (That is the intersection of the sphere with the plane through $x$ and $y$ and the centre of the sphere.). A natural way to parameterize arcs of great circles is as follows: given a point $x \in S^{2}$, a unit vector $u$ in the Euclidean space $\mathbb{R}^{3}$ such that $\langle x, u\rangle=0$, and a number $a \in[0, \pi]$, consider the path $c:[0, a] \longrightarrow S^{2}$ given by $c(t)=(\cos t) A+(\sin t) u$. We obtain

$$
d\left(c(t), c\left(t^{\prime}\right)\right) \leq\left|t-t^{\prime}\right| \quad \text { for all } t, t^{\prime} \in[0, a],
$$

and the image of $c$ is contained in the great circle where the vector subspace spanned by $x$ and $u$ meets $S^{2}$. The image of $c$ is said to be a minimal great arc, more precisely, the great arc with initial vector $u$ and joining $x$ to $c(a)$. Let $p=(0,0,1), q=$ $(-(\sqrt{3} / 3),(\sqrt{2} / 3),-(2 / 3)), \quad r=((\sqrt{3} / 3),(\sqrt{2} / 3),-(2 / 3))$, and $s=(0,-(2 \sqrt{2} / 3),(1 / 3))$. Since (Figure 2$)$,

$$
\begin{aligned}
\langle p, q\rangle & =\langle p, r\rangle=\langle s, q\rangle=\langle s, r\rangle \\
& =-\frac{2}{3} \text { and }\langle p, s\rangle=\langle q, r\rangle=\frac{1}{3},
\end{aligned}
$$

We obtain

$$
d(p, q)=d(p, r)=d(s, q)=d(s, r) \text { and } d(p, s)=d(q, r) \text {. }
$$

And, if we consider $C$ to be the major $\operatorname{arc}$ joining $p$ and $s$, we plainly see that

$$
\Delta_{(q, r)}(C)=D(p, q, r)=D(s, q, r) .
$$

Consider the vectors

$$
x=:\left(-\sqrt{\frac{3}{5}}, \sqrt{\frac{2}{5}}, 0\right) \text { and } y=:\left(\sqrt{\frac{3}{5}}, \sqrt{\frac{2}{5}}, 0\right),
$$

and the following paths 


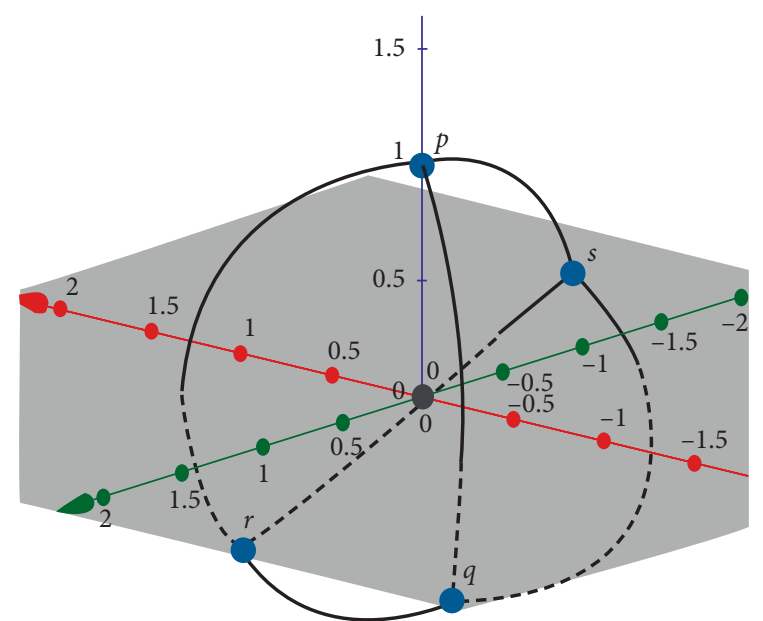

FIgURE 2: A counter example in three dimension.

$$
\begin{aligned}
& c:\left[0, \cos ^{-1}\left(-\frac{2}{3}\right)\right] \longrightarrow S^{2}, t \mapsto\left(-\sqrt{\frac{3}{5}} \sin t, \sqrt{\frac{2}{5}} \sin t, \cos t\right), \\
& c^{\prime}:\left[0, \cos ^{-1}\left(-\frac{2}{3}\right)\right] \longrightarrow S^{2}, t \mapsto\left(\sqrt{\frac{3}{5}} \sin t, \sqrt{\frac{2}{5}} \sin t, \cos t\right) .
\end{aligned}
$$

Clearly, the image of $c\left(c^{\prime}\right.$, resp. $)$ is the great arc with initial vector $x$ and joining $p$ to $q$ ( $y$ and joining $p$ to $r$, resp.). And,

$$
\langle x, y\rangle=-\frac{1}{5} \text { and }\langle x, s\rangle=\langle y, s\rangle=-\frac{4 \sqrt{5}}{15},
$$

implying that

$$
d(x, y)>d(q, r) \text { and } d(x, y)-d(q, r)>2(d(s, q)-d(s, x)) \text {. }
$$

Now, all of the conditions previously mentioned are furnished.

Remark 2. Retain the same notations as in the previous example. We emphasize that the major great $\operatorname{arc} C$ is not a geodesic segment in the sense of geodesic metric spaces. Indeed, an arc of a great circle on $S^{2}$ fails to be a geodesic segment as long as it is longer than half of the equator. However, if $S^{2}$ is seen as a two-dimensional manifold in the sense of differential geometry, $C$ is a geodesic and is geodesically convex.

\section{Data Availability}

No data were used to support this study.

\section{Conflicts of Interest}

The authors declare that there are no conflicts of interest regarding the publication of this paper.

\section{Acknowledgments}

This research was supported by the National Centre of Scientific and Technological Research grant.

\section{References}

[1] T. Sabar, M. Aamri, and A. Bassou, "Best proximity point of tricyclic contractions," Advances in Fixed Point Theory, vol. 7, no. 4, pp. 512-523, 2017.

[2] T. Sabar, A. Bassou, and M. Aamri, "Best proximity point for tricyclic contractions in (S) convex metric spaces," International Journal of Mathematical Analysis, vol. 12, no. 6, pp. 289-299, 2018.

[3] H. Busemann, The Geometry of Geodesics, Academic Press, New York, NY, USA, 1955.

[4] M. R. Bridson and A. Haefliger, Metric Spaces of Non-Positive Curvature, Springer-Verlag, Berlin, Germany, 1999.

[5] U. Kohlenbach, "Some logical metatheorems with application in functional analysis," Transactions of American Mathematical Society, vol. 357, no. 1, pp. 89-128, 2005.

[6] D. Burago, Y. Burago, and S. Ivanov, A Course in Metric Geometry, American Mathematical Society, Washington, D.C., USA, 2001. 